\title{
Understanding the Role of DISC1 in Psychiatric Disease and during Normal Development
}

\author{
Nicholas J. Brandon, ${ }^{1}$ J. Kirsty Millar, ${ }^{2}$ Carsten Korth, ${ }^{3}$ Hazel Sive, ${ }^{4,5,6}$ Karun K. Singh, ${ }^{7}$ and Akira Sawa ${ }^{8,9,10}$ \\ ${ }^{1}$ Discovery Neuroscience, Wyeth Research CN 8000, Princeton, New Jersey 08543, ${ }^{2}$ Medical Genetics Section, University of Edinburgh Centre for Molecular \\ Medicine and The Institute of Genetics \& Molecular Medicine, University of Edinburgh, Western General Hospital, Edinburgh EH4 2XU, United Kingdom, \\ ${ }^{3}$ Department of Neuropathology, University of Duesseldorf Medical School, 40225 Duesseldorf, Germany, ${ }^{4}$ Whitehead Institute for Biomedical Research, \\ ${ }^{5}$ Massachusetts Institute of Technology, and ${ }^{6}$ Stanley Center for Psychiatric Research, Broad Institute, Cambridge, Massachusetts 02142, ${ }^{7}$ Picower Institute \\ of Learning and Memory, Massachusetts Institute of Technology, Cambridge, Massachusetts 02139, and Departments of 8Psychiatry and Behavioral \\ Sciences and ${ }^{9}$ Neuroscience and ${ }^{10}$ Program in Cellular and Molecular Medicine, Johns Hopkins University School of Medicine, Baltimore, Maryland 21205
}

The biology of schizophrenia is complex with multiple hypotheses (dopamine, glutamate, neurodevelopmental) well supported to underlie the disease. Pathways centered on the risk factor "disrupted in schizophrenia 1" (DISC1) may be able to explain and unite these disparate hypotheses and will be the topic of this mini-symposium preview. Nearly a decade after its original identification at the center of a translocation breakpoint in a large Scottish family that was associated with major psychiatric disease, we are starting to obtain credible insights into its function and role in disease etiology. This preview will highlight a number of exciting areas of current DISC1 research that are revealing roles for DISC1 during normal brain development and also in the disease state. Together these different threads will provide a timely and exciting overview of the DISC1 field and its potential in furthering our understanding of psychiatric diseases and in developing new therapies.

\section{Introduction}

The disrupted in schizophrenia (DISC) gene locus was first identified as a risk factor for major mental illness through study of a large Scottish family in which a balanced translocation between chromosomes 1 and 11 cosegregates with schizophrenia, bipolar disorder, and recurrent major depression (Fig. 1) (St Clair et al., 1990; Millar et al., 2000). Statistical analysis indicates that inheritance of the translocation is causal and increases risk of developing one of these disorders by $\sim 50$-fold in comparison to the general population (Blackwood et al., 2001). This translocation simultaneously directly disrupts DISC1, a conventional protein coding gene, and DISC2, an antisense noncoding RNA gene on chromosome 1 (Millar et al., 2000). Since 2000, a number of complementary research efforts have started to show the importance and relevance of DISC1 for psychiatric disease. Subsequent genetic studies have confirmed that the DISC locus is involved in multiple psychiatric disorders and cognitive function, in several populations worldwide (Chubb et al., 2008; Jaaro-Peled, 2009). Efforts to model DISC1 disease biology in transgenic mice, and more recently in Drosophila and zebrafish, have been quite successful (Sawamura et al., 2008; Wang et al., 2008; Drerup et al., 2009; Kellendonk et al., 2009). In general DISC1 mutant mice, from a range of approaches, show behavioral and anatomical deficits that can be linked to psychiatric disease. In addition, the description of the "DISC1 interactome," a rich protein-protein interaction network around DISC1, has given the field a platform

Received July 13, 2009; accepted Aug. 7, 2009

We thank Yukiko Lema and Gianluca de Rienzo for assistance with the preparation of figures.

Correspondence should be addressed to Nicholas J.Brandon at the above address. E-mail: brandon@wyeth.com. D01:10.1523/JNEUROSCI.3355-09.2009

Copyright $\odot 2009$ Society for Neuroscience $\quad$ 0270-6474/09/2912768-08\$15.00/0 to interrogate processes and pathways that may be affected by DISC1 (Camargo et al., 2007).

This DISC1 mini-symposium will preview developments in this rapidly growing field. We will start with breakthroughs in understanding the molecular consequences of the original family translocation that might have critical repercussions for the field (Millar). Then switching gears a possible role for aberrant DISC1 protein multimerization and misassembly in a subset of sporadic disease cases will be described (Korth). This will segue into an analysis of the pathways affected by DISC1 at both a cellular and whole-animal level. The function of DISC-1 during normal development will be described (Sive), using the zebrafish as a tool. Zebrafish DISC-1 is an essential gene, required for forebrain development and GSK3/ $\beta$-catenin signaling. Moving into the mouse the DISC1-GSK3 pathway will then be shown to regulate the proliferation of neural progenitor cells in the developing murine cortex and adult dentate gyrus (Singh). Complementing this work, we will describe how DISC1 plays a key role at the centrosome (Sawa) and conclude with the identification of novel roles for DISC1 at synapses (Brandon). As the diversity of DISC1 function is elaborated through this session, we will pay close attention to the diseases to which DISC1 is a contributory factor and see the emergence of possible therapeutic approaches for DISC1 diseases (Wang et al., 2008).

\section{Understanding molecular lesions in DISC1 that underlie psychiatric illness}

It is critical that we understand how mutations in DISC1 can lead to molecular deficits that contribute to psychiatric illness. Obviously the original Scottish family with the balanced $t(1 ; 11)$ translocation provides the key molecular lesion to study, but recently 


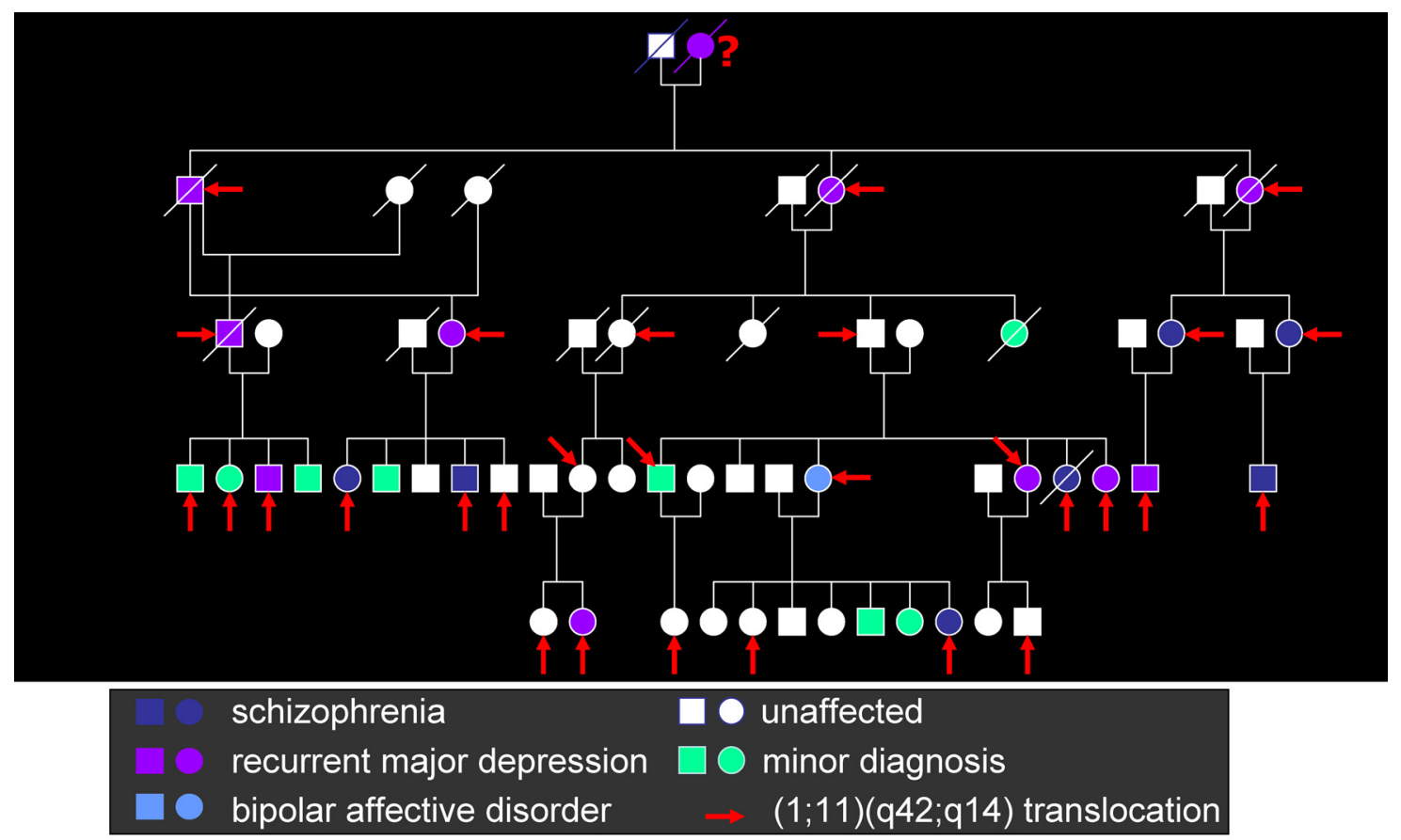

Adapted from Blackwood et al., 2001

Figure 1. Pedigree of the Scottish family showing inheritance of a balanced translocation $(1 ; 11)(q 42 ; q 14.3)$ and clinical diagnoses within the family [adapted with permission from Blackwood et al. (2001), their Fig. 1]. The translocation (carriers are indicted by a red arrow) is shown to be associated with a range of major psychiatric illnesses including schizophrenia, bipolar affective disorder, and major depression.

discovered rarer mutations in DISC1 and posttranslational processing events also need to be studied (see next section). The original cloning of the translocation breakpoints suggested that the disruption of DISC1 and DISC2 on chromosome 1 was critical with the apparent gene desert on chromosome 11 suggesting no other genes were involved (Millar et al., 2000). This has now changed with the identification of a novel, brain-expressed gene, named DISC1 Fusion Partner 1 (DISC1FP1), disrupted by the translocation on chromosome 11. With this finding it is possible that the disease mechanism is more complex than simple loss of normal function or gain of function with a truncated protein, because DISC1 and DISC1FP1 are located in the same orientation, and are predicted to form unique fusion transcripts and proteins as a result of the translocation (Zhou et al., 2008).

DISC1/DISC1FP1 fusion transcripts can be detected from both derived chromosomes in lymphoblastoid cell lines isolated from translocation carriers. Fusion transcripts from the derived 1 chromosome are relatively rare, while fusion transcripts from the derived 11 chromosome are relatively abundant. Fusions from the der 1 chromosome encode DISC1 amino acids 1-597 fused to 60 or 69 novel amino acids, depending on DISC1FP1 exon splicing. However, the open reading frame is followed by several splicing events which potentially mark these abnormal transcripts for nonsense-mediated decay, consistent with their apparent scarcity. When exogenously overexpressed, the aberrant DISC1 fusion proteins are strongly targeted to mitochondria where they induce morphological abnormalities. Fusions from the der 11 chromosome encode a C-terminal fragment of DISC1 corresponding to amino acids $669-854$. This open reading frame is preceded by several stop codons, and may therefore not be translated. Intriguingly however, if produced, this C-terminal fragment of DISC1 would correspond remarkably closely to the truncated portion of human DISC1 used to disrupt normal DISC1 function in mice by transgenic overexpression, resulting in a schizophrenia-like phenotype (Li et al., 2007). A third potential der 1-derived fusion transcript is under investigation. This fusion transcript encodes DISC1 amino acids 1-597, plus one additional amino acid. Like the other der 1-derived fusion transcripts, this transcript may be subject to nonsense-mediated decay due to the presence of splice sites after the stop codon. However, if translated, the resulting protein would be essentially identical to the truncated human DISC1 species used to generate transgenic mouse models of psychiatric illness (Hikida et al., 2007; Pletnikov et al., 2008). It also corresponds to the fragment of mouse DISC1 expressed from a truncated transgenic BAC (Shen et al., 2008) in a further model.

In addition to the translocation, several putative pathogenic mutations have been identified through sequencing DISC1 exons in patients (Song et al., 2008). As an example, R37W, was identified in a single patient with schizophrenia, but not in 5000 unaffected controls and is therefore classed as an "ultra-rare" mutation. Preliminary data show that this mutation is functional and enhances localization of DISC1 to mitochondria, where, like the abnormal DISC1 fusion proteins, it induces mitochondrial morphological abnormalities. Altogether these observations suggest that disease pathogenesis may involve abnormal compartmentalization of DISC1.

\section{Understanding the role of DISC1 misassembly in psychiatric illness}

As we reveal the fine molecular details of the original Scottish family translocation, we are also confronted by a major question of whether nonmutant, full-length DISC1 protein plays a role in sporadic forms of major psychiatric disease. The genetic data in a 


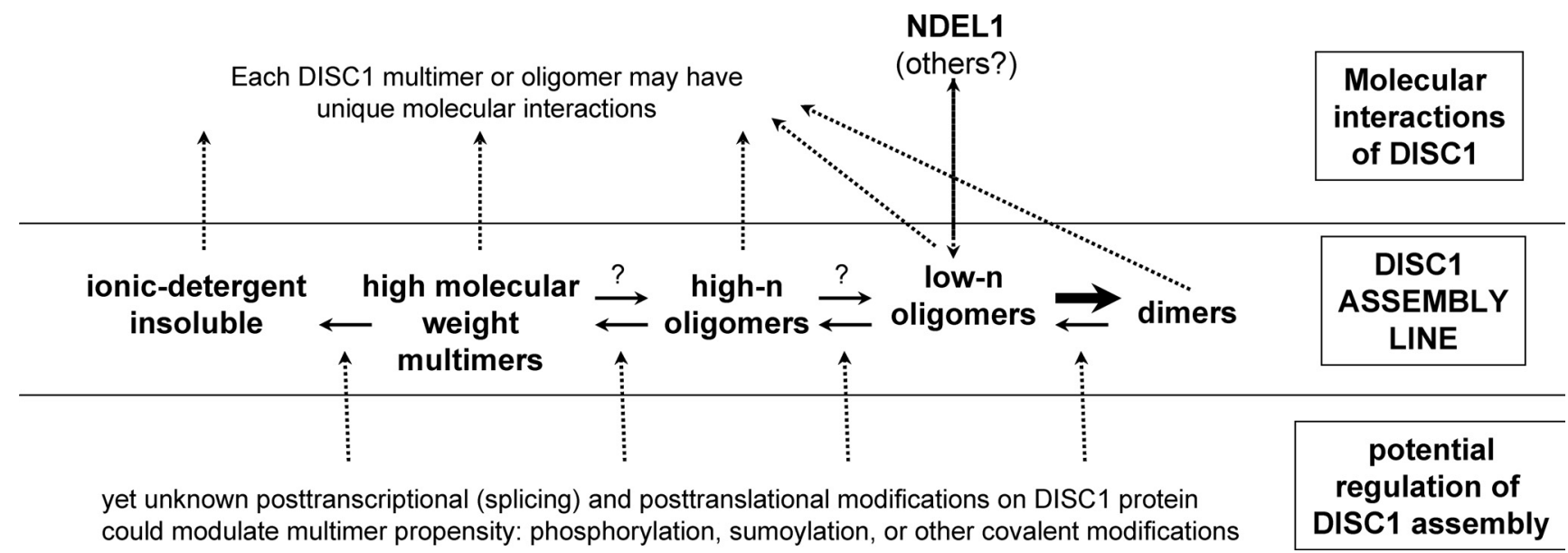

Figure 2. Diagram depicting a possible scenario how assembly and misassembly of DISC1 could regulate its function. Of note, experimental data that were used to generate this diagram were obtained in different in vivo and in vitro systems and were to a large extent obtained with (C-terminal) DISC1 fragments; the diagram's purpose is to generate testable hypotheses rather than to depict a complete picture of DISC1 physiology.

range of populations is fairly supportive to date of a role for DISC1 in the general population (Chubb et al., 2008). In addition there are well known precedents to how proteins that can be dysfunctional both as mutant proteins and as nonmutant proteins causing similar or even identical neuropsychiatric phenotypes. In Alzheimer's disease or Parkinson's disease, for example, proteins crucial to disease pathophysiology are mutant in familial forms of the disease, but the same proteins are also dysfunctional when they are posttranslationally modified (Prusiner, 2001). These posttranslational modifications include misfolding or misprocessing of specific proteins, or both (Prusiner, 2001; Taylor et al., 2002).

DISC1 is a cytosolic scaffold protein with four predicted coiled-coil domains; posttranslational modifications can theoretically include phosphorylation, sumoylation, multimerization, and proteolysis into active fragments. We investigated aberrant multimerization of DISC1 as one possible mechanism of how a posttranslational modification could affect its function. The middle part of DISC1 (residues 400-500) had been identified as a DISC1 self-association domain (Kamiya et al., 2005). The C-terminal part of DISC1 that is possibly deleted in the Scottish family harbors both a homomeric multimerization (residues 668-747) and a dimerization domain (residues 765-854), the concerted interaction between both being essential for ordered oligomerization to a functional C-terminal DISC1 domain in vitro (Leliveld et al., 2008, 2009). We found that distinct octamers but not dimers or high-molecular-weight multimers of the DISC1 C terminus bind to important interacting molecule nuclear-distribution element-like 1 (NDEL1), indicating that the DISC1-NDEL1 interaction depends on assembly of DISC1 to a defined quartenary structure (Fig. 2). When transiently overexpressed in human neuroblastoma cells, DISC1 segregates into aggresomes and reveals insolubility in the presence of ionic detergents whereas under same conditions, for example, NDEL1 remains soluble (Leliveld et al., 2008). Insoluble DISC1 was unable to interact with NDEL1, and in a subset of patients with psychiatric disease of mixed phenotypes insoluble DISC1 was identified to be associated with a subset of patients (Leliveld et al., 2008). Thus, aberrant multimerization into insoluble forms could be a dysfunctional correlate of DISC1 in a subset of cases with sporadic disease. From these studies, a group of sporadic disease cases could be related to DISC1 misassembly and thus constitute a larger group of brain disorders related to DISC1 dysfunction.

Higher multimerization propensity of DISC1 degradation products in DISC1 transiently overexpressing NLF cells has been described (Leliveld et al., 2008). At this point, it is unclear, however, whether DISC1 is proteolytically processed, in whether such fragments are physiologically functional. We have identified C-terminal fragments of DISC1 in human brains (Leliveld et al., 2009) and a transgenic mouse model of inducible expression of the C-terminal fragment has indicated a potential dominantnegative function for this fragment (Li et al., 2007). These findings suggest that DISC1 proteolytic fragments could regulate cellular events and potentially compete with full-length DISC1 for binding partners. Thus, posttranslational modifications and proteolysis of the DISC1 protein as well as existence of multiple splice forms of DISC1 add to a considerable complexity in how the nonmutant DISC1 gene is expressed. For defining future DISC1-based diagnostics and pharmacotherapy, it will be important to sort out DISC1 protein isoforms, their modifications and their interactors that ultimately are the smallest common denominator for causing a behavioral phenotype.

\section{Analysis of DISC1 function using the zebrafish tool}

We have begun to use the zebrafish as a tool to study the function of DISC1. In the study of mental health disorders, zebrafish do not display the complex behaviors present in humans, and cannot be used to recapitulate the disorder. We therefore draw the distinction between an animal "model," which recapitulates a human disorder, and a "tool," which does not, but nonetheless can be used to provide insight into the disorder. The zebrafish is an excellent tool for analysis of genes associated with mental health disorders, due to the ability to perform rapid loss- and gain-of-function assays, ability to assay function of human gene variants rapidly, and feasibility of small molecule screens (Blaker, 2009).

Conserved domains are present in the DISC1 gene in zebrafish and mammals. Zebrafish DISC1 is expressed maternally and zygotic expression is strongest in the developing CNS. To test the embryonic function of DISC1, we decreased zygotic gene function by injecting morpholino-modified antisense oligonucleotides (MOs) targeting splice sites. A DISC1 morpholino described as D1MO was directed against the splice donor site between exon 


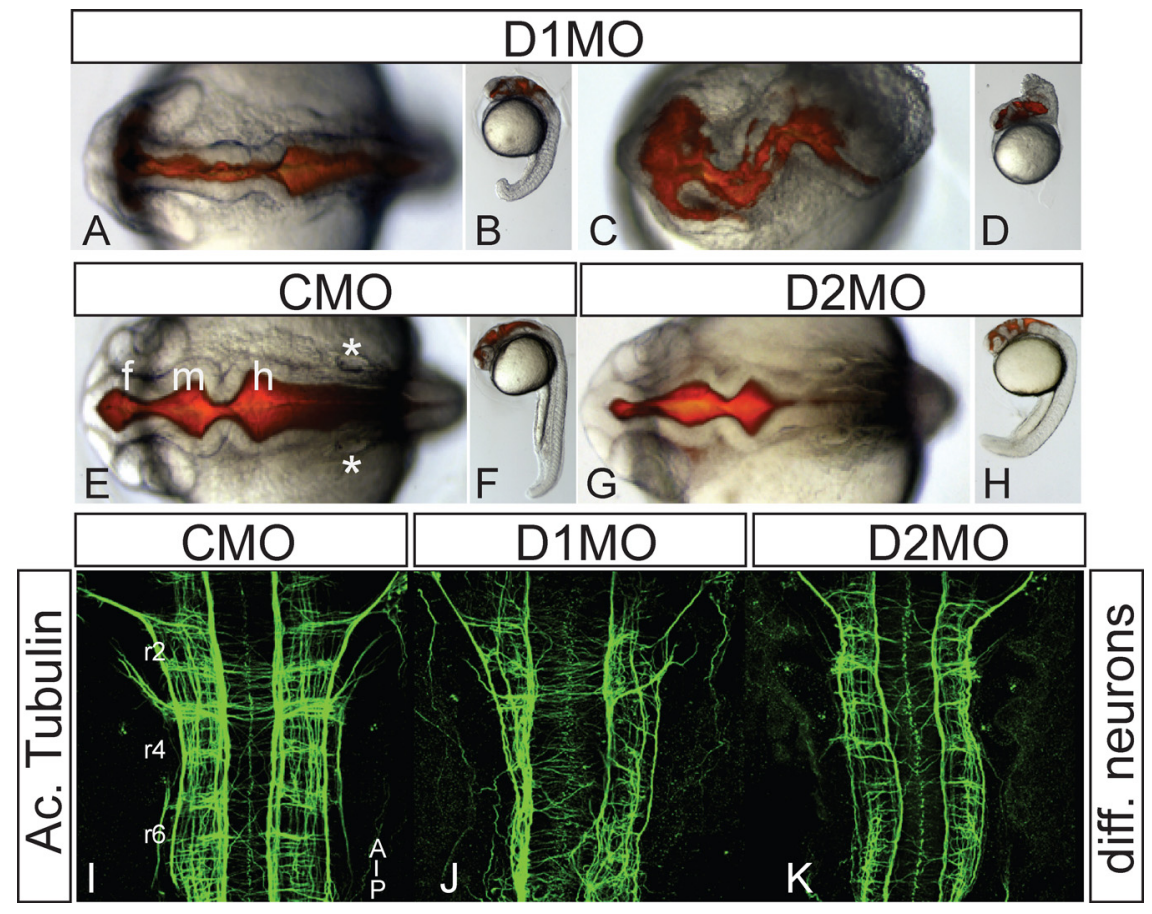

Figure 3. Knockdown of zebrafish DISC-1 protein function results in abnormal brain and impaired axonogenesis. Two antisense morpholino-modified oligonucleotides (M0), targeting splice sites, were injected at the one- to two-cell stage. The D1 M0 lies between exons 1 and 2, and ablates all functional DISC-1 protein, while the D2 MO lies between exons 8 and 9 and produces a truncated protein. Embryos were assayed at $24 \mathrm{~h}$ after fertilization in 4-6independent experiments, with at least 100 embryos per experiment. Brain ventricles were visualized after microinjecting a fluorescent dye, rhodamine-dextran, into the hindbrain ventricle of living anesthetized embryos. $A-D$, DISC1 morpholino 1 (D1M0)-injected embryos display one of two phenotypes. The D1M0 mild phenotype shows reduced brain ventricles with loss of normal shape $(\boldsymbol{A})$ and defects in the somites and head $(\boldsymbol{B})$. The D1M0 strong phenotype shows abnormal brain (C) and no tail formation (D). $\boldsymbol{E}, \boldsymbol{F}$, Control morpholino (CM0)-injected embryos display a wild-type phenotype. $\mathbf{G}, \boldsymbol{H}$, Embryos injected with the D2M0 display reduced hindbrain and midbrain ventricles $(\boldsymbol{G})$ and reduced and bent tail $(\boldsymbol{H})$. f, Forebrain; $\mathrm{m}$, midbrain; $\boldsymbol{h}$, hindbrain; asterisk, otic vesicles. $\boldsymbol{I}-\boldsymbol{K}$, Abnormal axon growth in D1 and D2 morphants, stained for differentiated neurons with anti-acetylated tubulin. Dorsal view of the hindbrain is shown. $I$, Control animals. J, Strongly reduced and disorganized axon growth in D1M0-injected embryos. $\boldsymbol{K}$, Reduction of axons in D2M0-injected embryos.

1 and intron 1/2, and ablates all normal DISC1 mRNA. D1MOinjected embryos ("morphants") show a very strong phenotype, apparent by early somitogenesis, including defects in forebrain formation and huge failure of axon outgrowth throughout the brain (Fig. 3A-D,J). In contrast, a second DISC1-targeted morpholino (D2MO) leads to aberrant splicing and truncation of the DISC-1 protein between exons 8 and 9, but gives a much less severe phenotype than D1 (Fig. 3G,H,K). This truncation recapitulates that seen in the human translocation associated with schizophrenia (Millar et al., 2000). Expression of this truncated protein in the brain of wildtype embryos results in embryos with a similar phenotype to those treated with the D2MO, suggesting that it may interfere with normal DISC-1 function.

We noticed that the gross phenotypes resulting from DISC1 and Wnt8b loss of function are similar, and showed that partial loss of function of each resulted in a strong phenotype, demonstrating synergy between these genes. Several additional lines of evidence indicate that DISC1 functions in the Wnt pathway. First, similar changes in forebrain gene expression are observed after loss of DISC1 or loss of Wnt8b function. Second, loss of DISC1 function decreases reporter gene expression in a Wnt-responsive reporter zebrafish line. Third, a Gsk $3 \beta$ inhibitor is able to rescue gene expression in DISC-1 loss of function morphants. Fourth, an inducible $\beta$-catenin fusion protein rescues the DISC-1 loss of function phenotype. Finally, we show that zebrafish and human DISC-1 function is conserved, since expression of the human gene can rescue DISC-1 morphants, and this rescue requires the Gsk3 $\beta$ binding domain present in DISC-1. These data are consistent with those obtained in late gestation mouse embryos that led to the demonstration of direct DISC- 1 and Gsk $3 \beta$ interaction (see next section) (Mao et al., 2009).

In conclusion, our data identify DISC- 1 as a crucial positive modulator of the canonical Wnt pathway, and confirm the efficacy of using zebrafish as a tool to explore the function of genes implicated in human mental health disorders.

\section{The role of DISC1 in progenitor cell proliferation}

Schizophrenia (SZ) is described as a neural development disorder since the age of onset primarily occurs in early adulthood. Moreover, it is thought that the pathology of SZ develops during prenatal and postnatal stages and that disrupted neural development at these time points leads to SZ in later years (Jaaro-Peled, 2009). Numerous studies have shown that DISC1 is highly expressed during brain development and plays vital roles in the growth of the embryonic and postnatal brain (Schurov et al., 2004; Chubb et al., 2008). This suggests that DISC1 contributes to psychiatric illness by affecting brain development and maturation.

A recent study from the Tsai laboratory revealed a novel role for DISC1 as an important regulator of embryonic and adult neurogenesis (Mao et al., 2009). The authors discovered that DISC1 is highly expressed in the embryonic ventricular/subventricular zones of the cortex where neural progenitor cells reside, suggesting DISC1 may regulate their proliferation and/or differentiation. To assess this, in utero electroporation was used to introduce DISC1 RNAi into neural progenitors in the developing neocortex. This resulted in significantly reduced proliferation of progenitor cells, concurrent with an increase in cell cycle exit and premature neuronal differentiation. Interestingly, overexpression of DISC1 in neural progenitors resulted in an opposite phenotype. These results suggest that the levels of DISC1 in cycling neural progenitor cells influences whether they remain as progenitors or differentiate into neurons. This study also found that DISC1 has a prominent role in adult neurogenesis. Knockdown of DISC1 using a lentivirus to deliver RNAi resulted in a decrease in the proliferation of adult progenitor cells in the dentate gyrus. Furthermore, this manipulation resulted in mice that displayed hyperlocomotion in a novel environment and increased immobility in the forced swimming test, both behavioral measures frequently observed in animal models displaying schizophrenic-like behavior.

In searching for mechanisms to explain how DISC1 regulates neurogenesis, Mao et al. (2009) discovered that downregulating levels of DISC1 abolished the ability of Wnt3a to stimulate proliferation of neural progenitor cells, suggesting DISC1 functions in the Wnt signaling pathway (Fig. 4). Interestingly, in contrast to 
the $\mathrm{C}$ terminus of DISC1 which binds NDELl1, the N-terminal region of DISC1 was found to directly bind and inhibit GSK3 $\beta$, thereby stabilizing $\beta$-catenin levels. Downregulating DISC1 expression decreased levels of $\beta$-catenin and TCF/ LEF gene-reporter activity, while overexpression of DISC1 has the opposite effects. Most striking was the observation that the in vitro and in vivo phenotypes due to DISC1 knockdown (both in the embryonic or adult brain) could be rescued by either overexpression of a degradationresistant form of $\beta$-catenin, or by pharmacological inhibition of GSK $3 \beta$. This suggests the cellular and behavioral results due to DISC1 knockdown can be alleviated by hyperactivation of the canonical Wnt signaling pathway. Together, these data argue that DISC1 is an important regulator of neural progenitor proliferation that acts by positively modulating canonical Wnt signaling via inhibition of GSK3 $\beta$ (Fig. 4).

\section{The role of DISC1 at the centrosome and microtubule networks}

Microtubule networks organized from the centrosome play important cellular roles in the brain, including progenitor cell proliferation, neuronal migration, and differentiation (Feng and Walsh, 2001; Badano et al., 2005). We originally identified a role for DISC1 in the microtubule/ centrosome cascade, in which we reported an interaction of DISC1 and NDEL1 and its significance for neurite outgrowth in neuroblastoma PC12 cells (Ozeki et al., 2003). More recently we have isolated additional DISC1 binding proteins associated with the centrosome, which include pericentriolar material 1 (PCM1) and Bardet-Biedl syndrome proteins (BBSs) (Kamiya et al., 2008) (Fig. 5). BBS4 is known as an adaptor of the p150glued subunit of the dynein motor complex and to recruit PCM1 to the centrosome (Kim et al., 2004).

Many reports have now consistently indicated that the C-terminal domain of DISC1, which is lost in the Scottish mutation, play a key role in interactions with most of these DISC1 interacting centrosomal proteins, facilitating the centrosomal association (Ozeki et al., 2003; Brandon et al., 2004; Millar et al., 2005a; Shimizu et al., 2008) (Fig. 5). In addition, DISC1 contains a self-association domain in the middle portion of the protein (Kamiya et al., 2005). This domain may allow for the putative C-terminal truncated DISC1 to function in a dominant-negative manner by redistributing wild-type DISC1 through self-association and by dissociating the DISC1-motor complex from the centrosome. Consequently, either depletion of endogenous DISC1 or expression of a C-terminal truncated DISC1 impairs neurite outgrowth
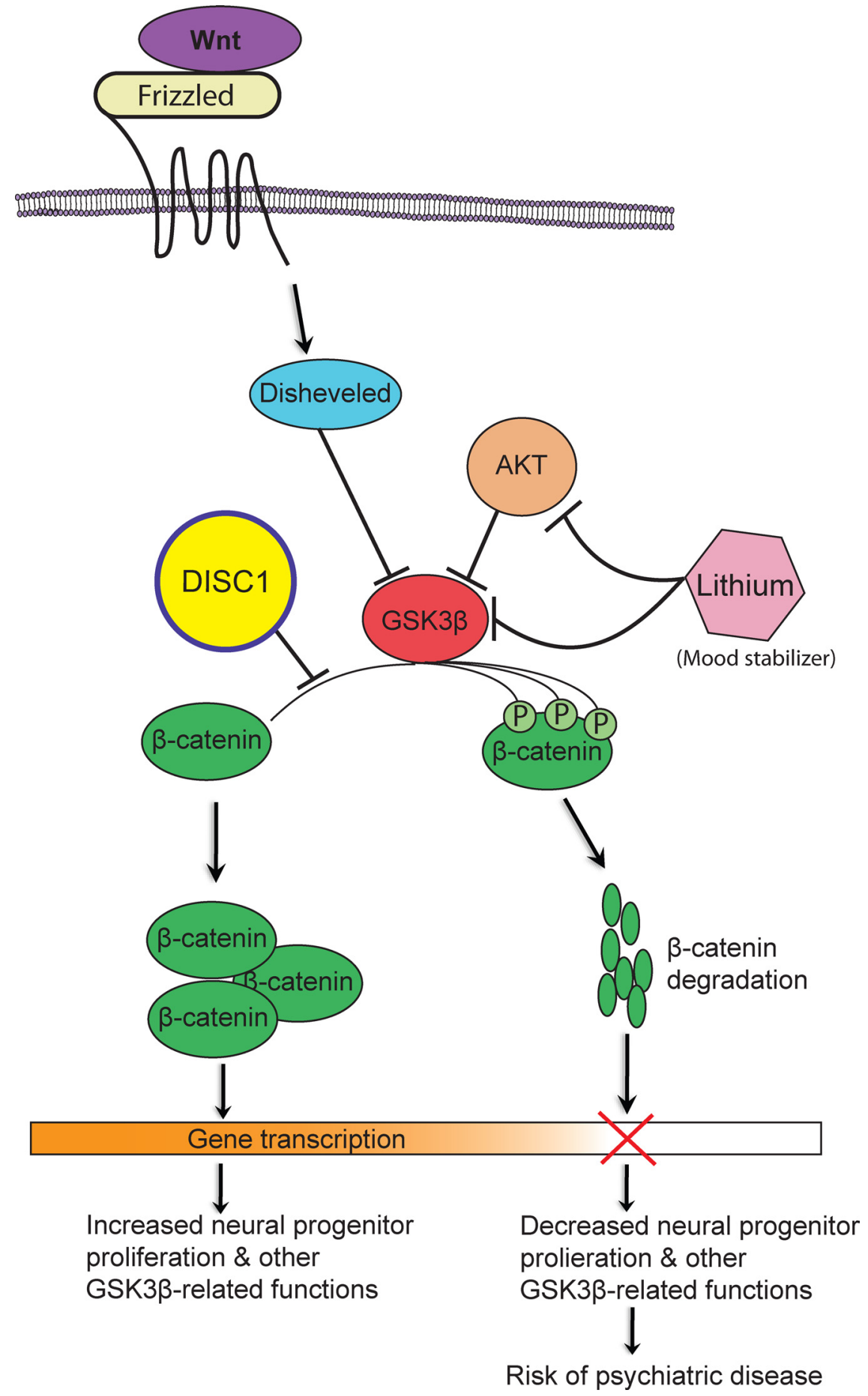

Figure 4. DISC1 regulates neurogenesis via Wnt/ $\beta$-catenin signaling. During canonical Wnt signaling, $\beta$-catenin levels are kept low in the cytosol due to GSK3 $\beta$-mediated phosphorylation which targets $\beta$-catenin for degradation. In the presence of Wnt ligands, the receptor complex and downstream signaling machinery is engaged, leading to increased accumulation of $\beta$-catenin and transcription of Wnt-dependent genes. In embryonic and adult neural progenitors, DISC1 directly binds and inhibits the function of GSK3 $\beta$, thereby increasing cytosolic $\beta$-catenin concentration and functioning as a positive regulator of Wnt signaling. This function of DISC1 is similar to Akt and Lithium (used to treat mood disorders), which can also inhibit GSK3 $\beta$, suggesting this pathway plays an important role in psychiatric disease.

in vitro, proper development of the cerebral cortex in vivo, and elicits behavioral deficits when expressed in mouse models (Kamiya et al., 2005; Hikida et al., 2007; Li et al., 2007; Pletnikov et al., 2008; Shen et al., 2008; Wang et al., 2008).

The importance of the DISC1 protein complex associated with microtubule motors and the centrosome in schizophrenia 


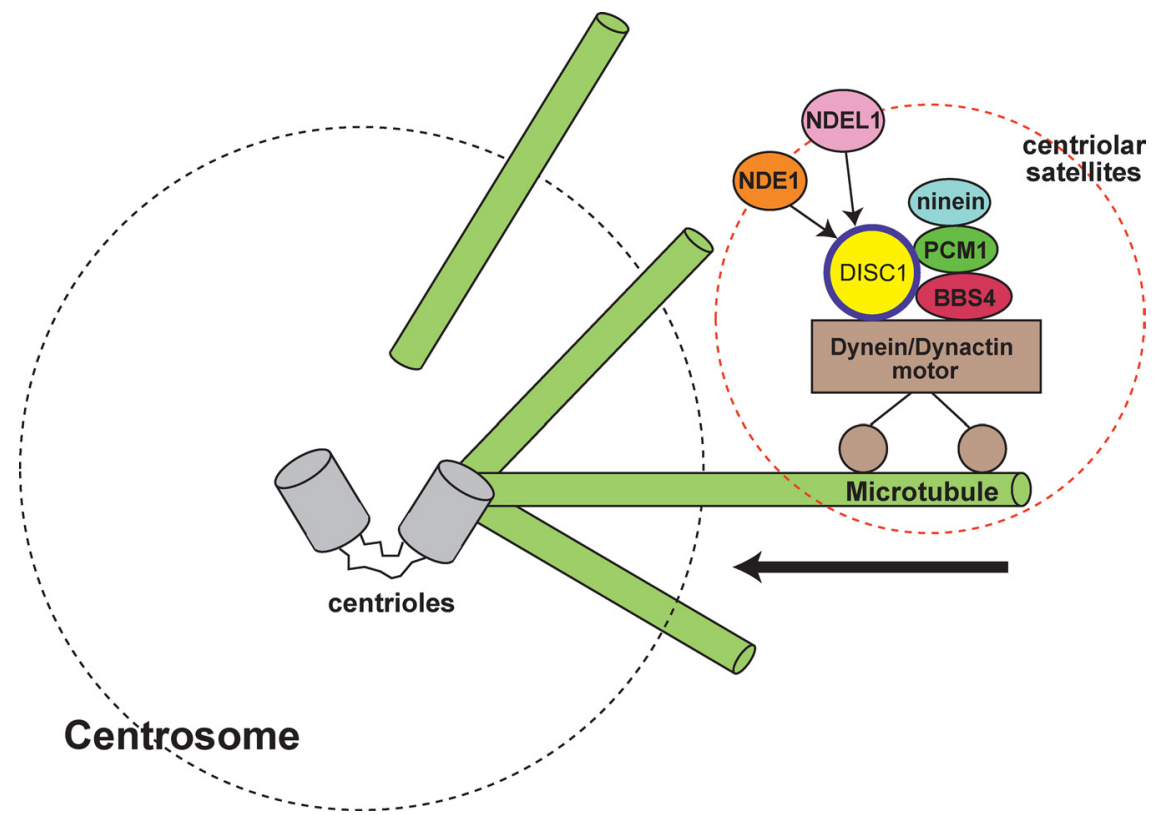

Figure 5. DISC1 at the centrosome. DISC1 is now known to bind to a number of proteins localized to the centrosome including nudE nuclear distribution gene E homolog (A. nidulans)-like 1 (NDEL1), pericentriolar material 1 (PCM1), and Bardet-Biedl syndrome proteins (BBSS). DISC1 play a role in anchoring these molecules in association with the dynein motor complex and centrosome, regulating microtubule organization. Of note, several of these DISC1 interactors at the centrosome are promising genetic risk factors for major mental illnesses in their own right.

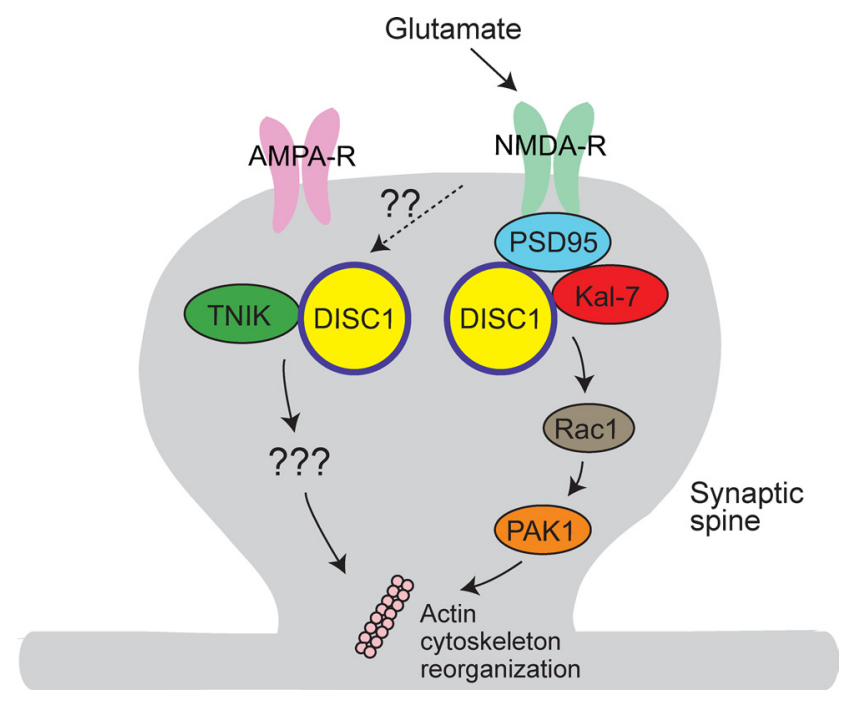

Figure 6. Model of DISC1 at the synapse. Multiple pieces of evidence show that DISC1 is a component of the postsynaptic density (PSDs) of excitatory synapses and regulates their form and function. It is likely that the effects of DISC1 are mediated through a range of protein interaction partners. The function of complexes of DISC1 and PSD proteins Kalirin-7 (kal-7) and Traf- and nck-interacting kinase (TNIK) are currently being elaborated. Kal-7 and TNIK both regulate the actin cytoskeleton, but it is currently unknown whether these two complexes act in a common pathway or independently of each other.

has been supported by two lines of genetic findings. First, genes encoding for these motor and centrosomal proteins have been reported in genetic association studies. Thus far, the most reproducible association has been in the $P C M 1$ gene. Initially, Gurling et al. (2006) reported that PCM1 is implicated in susceptibility to schizophrenia and is associated with orbitofrontal gray matter volumetric deficits. Genetic association of PCM1 with schizophrenia has since been reproduced in several sample sets (Datta et al., 2008). Furthermore, two groups including our own have reported nonsynonymous mu- tations (missense and nonsense) in the $P C M 1$ gene, in association with schizophrenia (Datta et al., 2008; Kamiya et al., 2008). The second line of evidence supporting the connection of DISC1 and motors/centrosome with schizophrenia are reports in which nonsynonymous genetic variations in DISC1 associated with schizophrenia, such as S704C and L607F (Hodgkinson et al., 2004; Callicott et al., 2005), seem to have a functional influence on protein interactions of DISC1 and motor/centrosomal proteins. We have been able to show that NDEL1 and NDE1 share the same DISC1 binding domain, and opposing binding patterns of NDEL1 and NDE1 to S704 versus C704 are observed (Kamiya et al., 2006; Burdick et al., 2008).

Together, although further genetic and neuropathological support are needed to lead to a firm conclusion, various lines of evidence have supported that a subsection of the "DISC1 interactome" associated with the centrosome and microtubule motors is likely to play a role in the pathology of schizophrenia.

\section{A role for DISC1 at the synapse}

In postmortem studies of schizophrenia patients, a consistent observation has been a reduction in spine density on cortical pyramidal neurons (Glantz and Lewis, 2000). Dendritic spines contain the postsynaptic compartment of the majority of excitatory synapses in the brain and their morphology is inextricably linked to neuronal activation and synaptic plasticity (Blanpied and Ehlers, 2004; Tada and Sheng, 2006). Accumulating data suggest DISC1 is localized at synapses, but its function(s) here remains poorly understood. The initial indication of a synaptic form of DISC1 came from an electron microscopic study of human neocortex that showed that DISC1 is found at asymmetric synapses principally on the postsynaptic side (Kirkpatrick et al., 2006). The ever-increasing number of DISC1 mouse models has added to the evidence for a role at the synapse, in particular through analysis of the neuronal architecture of some of these strains of mice. For example C57BL/6J mice, which carry a 25 bp deletion in Disc1 exon 6, produce a truncated Discl protein mimicking the Scottish family (Kvajo et al., 2008). These mice have significant decreases in synaptic spines in the dentate gyrus, and impairments in working memory as a consequence (Kvajo et al., 2008). While depletion of Discl in the dentate gyrus by shRNA in adult C57BL/6 mice led to accelerated formation of dendritic spines in newborn neurons with both glutamatergic and GABAergic synapses involved as measured functionally by electrophysiology (Duan et al., 2007).

These observations provide good support for a bioinformatics analysis of DISC1 interactions which strongly suggested that DISC1 is likely to be a key component of the postsynaptic density and a player in regulating synaptic plasticity (Camargo et al., 2007). This study was driven by a series of iterative yeast two hybrid screens which culminated in the "DISC1 interactome," a highly integrated network of protein-protein interactions around DISC1. We have approached synaptic DISC1 by understanding its protein environment at the synapse and so have re- 
lied on the DISC1 interactome for guidance (Camargo et al., 2007). As mentioned one of cellular processes enriched in this protein network was actin cytoskeleton regulation. The actin cytoskeleton is critical for regulating spine formation, which underlies synaptic plasticity (Sekino et al., 2007; Cingolani and Goda, 2008). To understand whether DISC1 plays a role in spine function, through regulating the actin cytoskeleton, we focused our efforts on DISC1 interacting partners that have been implicated in this process. The first interaction we have started to explore is between DISC1 and kalirin-7 (kal-7). Kal-7 was a very attractive target as it is reduced in schizophrenic brain (Hill et al., 2006). Kal-7 is a Rac1-GTP exchange factor (GEF) that activates Rac1 and is known to be downstream of a number of key signaling pathways at the synapse. Multiple lines of evidence now support a critical role for kal-7 in regulating spine morphology and underlying synaptic plasticity (Penzes and Jones, 2008). For example NMDAR activation has been shown to enhance kal-7 activity and in turn activate racl signaling. This in turn leads to increased spine size, an increase in GluR1 content in the spine and increased synaptic strength (Xie et al., 2007). It is likely that these events are driven by racl regulation of the actin cytoskeleton. We have recently characterized a DISC1-Kal-7-rac1 complex and shown that DISC1 is critical for regulating kal-7 activity and in turn the regulation of spine form and function (A. Hayashi and A. Sawa, unpublished results) (Fig. 6). In parallel we have analyzed the role of DISC1 in regulating the activity of a member of the Sterile-20 (Ste20) group of kinases known as Traf- and nckinteracting kinase (TNIK) (Q. Wang and N. Brandon, unpublished results). TNIK is an attractive partner to characterize as it has been recently linked to altered cortical function in schizophrenic patients and at the cellular level has been linked to actin cytoskeleton regulation (Fu et al., 1999; Potkin et al., 2009). The challenge now is to understand how DISC1 regulate both the kal-7 and TNIK pathways and how this is integrated with additional binding partner pathways at the synapse (Fig. 6). It is clear, though, that DISC1 is building up a curriculum vitae as a major player at the postsynaptic density.

\section{Discussion and perspective}

Emerging evidence suggests that DISC1 plays multiple key roles in normal brain development, while a number of mechanisms from the gene to aberrant protein processing may contribute to psychiatric disease etiology. The complexity of DISC1 biology should be of no surprise due to complexity at the transcriptional and translational levels and in the number of proteins to which DISC1 binds. As the pathways regulated by DISC1 emerge, for example cAMP via PDE4 previously (Millar et al., 2005b) and the wnt pathways via GSK3 herein, it is clear that therapeutic opportunities to reverse DISC1-related deficits may arise. Further study of these disease mechanisms and pathways through ever-increasing refined animal models and cell biology approaches is likely to bring this hope to a reality.

\section{References}

Badano JL, Teslovich TM, Katsanis N (2005) The centrosome in human genetic disease. Nat Rev Genet 6:194-205.

Blackwood DH, Fordyce A, Walker MT, St Clair DM, Porteous DJ, Muir WJ (2001) Schizophrenia and affective disorders-cosegregation with a translocation at chromosome 1q42 that directly disrupts brain-expressed genes: clinical and P300 findings in a family. Am J Hum Genet 69:428-433.

Blaker A, DeRienzo G, Sive H (2009) Zebrafish as a tool to study autism. In: Autism spectrum disorders (Amaral DG, Dawson G, Geschwind DH, eds), in press. Oxford: Oxford UP.
Blanpied TA, Ehlers MD (2004) Microanatomy of dendritic spines: emerg ing principles of synaptic pathology in psychiatric and neurological disease. Biol Psychiatry 55:1121-1127.

Brandon NJ, Handford EJ, Schurov I, Rain JC, Pelling M, Duran-Jimeniz B, Camargo LM, Oliver KR, Beher D, Shearman MS, Whiting PJ (2004) Disrupted in Schizophrenia 1 and Nudel form a neurodevelopmentally regulated protein complex: implications for schizophrenia and other major neurological disorders. Mol Cell Neurosci 25:42-55.

Burdick KE, Kamiya A, Hodgkinson CA, Lencz T, DeRosse P, Ishizuka K, Elashvili S, Arai H, Goldman D, Sawa A, Malhotra AK (2008) Elucidating the relationship between DISC1, NDEL1 and NDE1 and the risk for schizophrenia: evidence of epistasis and competitive binding. Hum Mol Genet 17:2462-2473.

Callicott JH, Straub RE, Pezawas L, Egan MF, Mattay VS, Hariri AR, Verchinski BA, Meyer-Lindenberg A, Balkissoon R, Kolachana B, Goldberg TE, Weinberger DR (2005) Variation in DISC1 affects hippocampal structure and function and increases risk for schizophrenia. Proc Natl Acad Sci U S A 102:8627-8632.

Camargo LM, Collura V, Rain JC, Mizuguchi K, Hermjakob H, Kerrien S, Bonnert TP, Whiting PJ, Brandon NJ (2007) Disrupted in Schizophrenia 1 Interactome: evidence for the close connectivity of risk genes and a potential synaptic basis for schizophrenia. Mol Psychiatry 12:74-86.

Chubb JE, Bradshaw NJ, Soares DC, Porteous DJ, Millar JK (2008) The DISC locus in psychiatric illness. Mol Psychiatry 13:36-64.

Cingolani LA, Goda Y (2008) Actin in action: the interplay between the actin cytoskeleton and synaptic efficacy. Nat Rev Neurosci 9:344-356.

Datta SR, McQuillin A, Rizig M, Blaveri E, Thirumalai S, Kalsi G, Lawrence J, Bass NJ, Puri V, Choudhury K, Pimm J, Crombie C, Fraser G, Walker N, Curtis D, Zvelebil M, Pereira A, Kandaswamy R, St Clair D, Gurling HM (2008) A threonine to isoleucine missense mutation in the pericentriolar material 1 gene is strongly associated with schizophrenia. Mol Psychiatry. Advance online publication. Retrieved Dec. 2, 2008. doi:10.1038/mp.2008.128.

Drerup CM, Wiora HM, Topczewski J, Morris JA (2009) Discl regulates foxd 3 and sox10 expression, affecting neural crest migration and differentiation. Development. 136:2623-2632.

Duan X, Chang JH, Ge S, Faulkner RL, Kim JY, Kitabatake Y, Liu XB, Yang CH, Jordan JD, Ma DK, Liu CY, Ganesan S, Cheng HJ, Ming GL, Lu B, Song H (2007) Disrupted-In-Schizophrenia 1 regulates integration of newly generated neurons in the adult brain. Cell 130:1146-1158.

Feng Y, Walsh CA (2001) Protein-protein interactions, cytoskeletal regulation and neuronal migration. Nat Rev Neurosci 2:408-416.

Fu CA, Shen M, Huang BC, Lasaga J, Payan DG, Luo Y (1999) TNIK, a novel member of the germinal center kinase family that activates the c-Jun $\mathrm{N}$-terminal kinase pathway and regulates the cytoskeleton. J Biol Chem 274:30729-30737.

Glantz LA, Lewis DA (2000) Decreased dendritic spine density on prefrontal cortical pyramidal neurons in schizophrenia. Arch Gen Psychiatry 57:65-73.

Gurling HM, Critchley H, Datta SR, McQuillin A, Blaveri E, Thirumalai S, Pimm J, Krasucki R, Kalsi G, Quested D, Lawrence J, Bass N, Choudhury K, Puri V, O’Daly O, Curtis D, Blackwood D, Muir W, Malhotra AK, Buchanan RW, Good CD, Frackowiak RS, Dolan RJ (2006) Genetic association and brain morphology studies and the chromosome $8 \mathrm{p} 22$ pericentriolar material 1 (PCM1) gene in susceptibility to schizophrenia. Arch Gen Psychiatry 63:844-854.

Hikida T, Jaaro-Peled H, Seshadri S, Oishi K, Hookway C, Kong S, Wu D, Xue R, Andradé M, Tankou S, Mori S, Gallagher M, Ishizuka K, Pletnikov M, Kida S, Sawa A (2007) Dominant-negative DISC1 transgenic mice display schizophrenia-associated phenotypes detected by measures translatable to humans. Proc Natl Acad Sci U S A 104:14501-14506.

Hill JJ, Hashimoto T, Lewis DA (2006) Molecular mechanisms contributing to dendritic spine alterations in the prefrontal cortex of subjects with schizophrenia. Mol Psychiatry 11:557-566.

Hodgkinson CA, Goldman D, Jaeger J, Persaud S, Kane JM, Lipsky RH, Malhotra AK (2004) Disrupted in schizophrenia 1 (DISC1): association with schizophrenia, schizoaffective disorder, and bipolar disorder. Am J Hum Genet 75:862-872.

Jaaro-Peled H, Hayashi-Takagi A, Seshadri S, Kamiya A, Brandon NJ, Sawa A (2009) Neurodevelopmental mechanisms of schizophrenia: understanding disturbed postnatal brain maturation through neuregulin-1-ERBB4 and DISC1. Trends Neurosci 32:485-495.

Kamiya A, Kubo K, Tomoda T, Takaki M, Youn R, Ozeki Y, Sawamura N, 
Park U, Kudo C, Okawa M, Ross CA, Hatten ME, Nakajima K, Sawa A (2005) A schizophrenia-associated mutation of DISC1 perturbs cerebral cortex development. Nat Cell Biol 7:1167-1178.

Kamiya A, Tomoda T, Chang J, Takaki M, Zhan C, Morita M, Cascio MB, Elashvili S, Koizumi H, Takanezawa Y, Dickerson F, Yolken R, Arai H, Sawa A (2006) DISC1-NDEL1/NUDEL protein interaction, an essential component for neurite outgrowth, is modulated by genetic variations of DISC1. Hum Mol Genet 15:3313-3323.

Kamiya A, Tan PL, Kubo K, Engelhard C, Ishizuka K, Kubo A, Tsukita S, Pulver AE, Nakajima K, Cascella NG, Katsanis N, Sawa A (2008) Recruitment of PCM1 to the centrosome by the cooperative action of DISC1 and BBS4: a candidate for psychiatric illnesses. Arch Gen Psychiatry 65:996-1006.

Kellendonk C, Simpson EH, Kandel ER (2009) Modeling cognitive endophenotypes of schizophrenia in mice. Trends Neurosci 32:347-358.

Kim JC, Badano JL, Sibold S, Esmail MA, Hill J, Hoskins BE, Leitch CC, Venner K, Ansley SJ, Ross AJ, Leroux MR, Katsanis N, Beales PL (2004) The Bardet-Biedl protein BBS4 targets cargo to the pericentriolar region and is required for microtubule anchoring and cell cycle progression. Nat Genet 36:462-470

Kirkpatrick B, Xu L, Cascella N, Ozeki Y, Sawa A, Roberts RC (2006) DISC1 immunoreactivity at the light and ultrastructural level in the human neocortex. J Comp Neurol 497:436-450.

Kvajo M, McKellar H, Arguello PA, Drew LJ, Moore H, MacDermott AB, Karayiorgou M, Gogos JA (2008) A mutation in mouse Disc1 that models a schizophrenia risk allele leads to specific alterations in neuronal architecture and cognition. Proc Natl Acad Sci U S A 105:7076-7081.

Leliveld SR, Bader V, Hendriks P, Prikulis I, Sajnani G, Requena JR, Korth C (2008) Insolubility of disrupted-in-schizophrenia 1 disrupts oligomerdependent interactions with nuclear distribution element 1 and is associated with sporadic mental disease. J Neurosci 28:3839-3845.

Leliveld SR, Hendriks P, Michel M, Sajnani G, Bader V, Trossbach S, Prikulis I, Hartmann R, Jonas E, Willbold D, Requena JR, Korth C (2009) Oligomer assembly of the C-terminal DISC1 domain (640-854) is controlled by self-association motifs and disease-associated polymorphism S704C. Biochemistry 48:7746-7755.

Li W, Zhou Y, Jentsch JD, Brown RA, Tian X, Ehninger D, Hennah W, Peltonen L, Lönnqvist J, Huttunen MO, Kaprio J, Trachtenberg JT, Silva AJ, Cannon TD (2007) Specific developmental disruption of disrupted-inschizophrenia-1 function results in schizophrenia-related phenotypes in mice. Proc Natl Acad Sci U S A 104:18280-18285.

Mao Y, Ge X, Frank CL, Madison JM, Koehler AN, Doud MK, Tassa C, Berry EM, Soda T, Singh KK, Biechele T, Petryshen TL, Moon RT, Haggarty SJ, Tsai LH (2009) Disrupted in schizophrenia 1 regulates neuronal progenitor proliferation via modulation of GSK3beta/beta-catenin signaling. Cell 136:1017-1031.

Millar JK, Wilson-Annan JC, Anderson S, Christie S, Taylor MS, Semple CA, Devon RS, Clair DM, Muir WJ, Blackwood DH, Porteous DJ (2000) Disruption of two novel genes by a translocation co-segregating with schizophrenia. Hum Mol Genet 9:1415-1423.

Millar JK, James R, Christie S, Porteous DJ (2005a) Disrupted in schizophrenia 1 (DISC1): subcellular targeting and induction of ring mitochondria. Mol Cell Neurosci 30:477-484.

Millar JK, Pickard BS, Mackie S, James R, Christie S, Buchanan SR, Malloy MP, Chubb JE, Huston E, Baillie GS, Thomson PA, Hill EV, Brandon NJ, Rain JC, Camargo LM, Whiting PJ, Houslay MD, Blackwood DH, Muir WJ, Porteous DJ (2005b) DISC1 and PDE4B are interacting genetic fac- tors in schizophrenia that regulate cAMP signaling. Science 310:11871191.

Ozeki Y, Tomoda T, Kleiderlein J, Kamiya A, Bord L, Fujii K, Okawa M, Yamada N, Hatten ME, Snyder SH, Ross CA, Sawa A (2003) Disruptedin-Schizophrenia-1 (DISC-1): mutant truncation prevents binding to NudE-like (NUDEL) and inhibits neurite outgrowth. Proc Natl Acad Sci U S A 100:289-294.

Penzes P, Jones KA (2008) Dendritic spine dynamics - a key role for kalirin-7. Trends Neurosci 31:419-427.

Pletnikov MV, Ayhan Y, Nikolskaia O, Xu Y, Ovanesov MV, Huang H, Mori S, Moran TH, Ross CA (2008) Inducible expression of mutant human DISC1 in mice is associated with brain and behavioral abnormalities reminiscent of schizophrenia. Mol Psychiatry 13:173-186, 115.

Potkin SG, Turner JA, Guffanti G, Lakatos A, Fallon JH, Nguyen DD, Mathalon D, Ford J, Lauriello J, Macciardi F (2009) A genome-wide association study of schizophrenia using brain activation as a quantitative phenotype. Schizophr Bull 35:96-108.

Prusiner SB (2001) Shattuck lecture-neurodegenerative diseases and prions. N Engl J Med 344:1516-1526.

Sawamura N, Ando T, Maruyama Y, Fujimuro M, Mochizuki H, Honjo K, Shimoda M, Toda H, Sawamura-Yamamoto T, Makuch LA, Hayashi A, Ishizuka K, Cascella NG, Kamiya A, Ishida N, Tomoda T, Hai T, Furukubo-Tokunaga K, Sawa A (2008) Nuclear DISC1 regulates CREmediated gene transcription and sleep homeostasis in the fruit fly. Mol Psychiatry 13:1138-1148, 1069.

Schurov IL, Handford EJ, Brandon NJ, Whiting PJ (2004) Expression of disrupted in schizophrenia 1 (DISC1) protein in the adult and developing mouse brain indicates its role in neurodevelopment. Mol Psychiatry 9:1100-1110.

Sekino Y, Kojima N, Shirao T (2007) Role of actin cytoskeleton in dendritic spine morphogenesis. Neurochem Int 51:92-104.

Shen S, Lang B, Nakamoto C, Zhang F, Pu J, Kuan SL, Chatzi C, He S, Mackie I, Brandon NJ, Marquis KL, Day M, Hurko O, McCaig CD, Riedel G, St Clair D (2008) Schizophrenia-related neural and behavioral phenotypes in transgenic mice expressing truncated Disc1. J Neurosci 28:10893-10904.

Shimizu S, Matsuzaki S, Hattori T, Kumamoto N, Miyoshi K, Katayama T, Tohyama M (2008) DISC1-kendrin interaction is involved in centrosomal microtubule network formation. Biochem Biophys Res Commun 377:1051-1056.

Song W, Li W, Feng J, Heston LL, Scaringe WA, Sommer SS (2008) Identification of high risk DISC1 structural variants with a $2 \%$ attributable risk for schizophrenia. Biochem Biophys Res Commun 367:700-706.

St Clair D, Blackwood D, Muir W, Carothers A, Walker M, Spowart G, Gosden C, Evans HJ (1990) Association within a family of a balanced autosomal translocation with major mental illness. Lancet 336:13-16.

Tada T, Sheng M (2006) Molecular mechanisms of dendritic spine morphogenesis. Curr Opin Neurobiol 16:95-101.

Taylor JP, Hardy J, Fischbeck KH (2002) Toxic proteins in neurodegenerative disease. Science 296:1991-1995.

Wang Q, Jaaro-Peled H, Sawa A, Brandon NJ (2008) How has DISC1 enabled drug discovery? Mol Cell Neurosci 37:187-195.

Xie Z, Srivastava DP, Photowala H, Kai L, Cahill ME, Woolfrey KM, Shum CY, Surmeier DJ, Penzes P (2007) Kalirin-7 controls activity-dependent structural and functional plasticity of dendritic spines. Neuron 56:640-656.

Zhou X, Geyer MA, Kelsoe JR (2008) Does disrupted-in-schizophrenia (DISC1) generate fusion transcripts? Mol Psychiatry 13:361-363. 